\title{
Unusual Attachment of Left Atrial Myxoma: Role of Transesophageal Echocardiography
}

\author{
${ }^{1}$ Neeti Makhija, ${ }^{2}$ Kalpna Irpachi, ${ }^{3}$ Ujjwal K Chowdhury, ${ }^{4}$ Usha Kiran
}

\begin{abstract}
Left atrial myxomas are usually attached to the interatrial septum. This case highlights the unusual site of attachment of left atrial myxoma. The site of attachment was via a broad stalk at the junction of mitral valve annulus close to anterior mitral leaflet and adjacent left atrial wall. Transesophageal echocardiography helped in the detection of site of attachment as well as in postoperative evaluation.
\end{abstract}

Keywords: Left atrial mass, Left atrial myxoma, TEE, TTE.

How to cite this article: Makhija N, Irpachi K, Chowdhury UK, Kiran U. Unusual Attachment of Left Atrial Myxoma: Role of Transesophageal Echocardiography. J Perioper Echocardiogr 2016;4(1):30-33.

\section{Source of support: Nil \\ Conflict of interest: None}

\section{INTRODUCTION}

Atrial myxomas are the commonest primary tumors of the heart and mostly occur in the left atrium (LA). They are mostly attached to the interatrial septum usually via a stalk. We present a case where left atrial myxoma was attached at an unusual site. The site of attachment was via a broad stalk at the junction of mitral valve annulus close to anterior mitral leaflet (AML) and adjacent LA wall. Transesophageal echocardiography (TEE) helped in the detection of site of attachment as well as in postoperative evaluation.

\section{CASE REPORT}

A 44-year-old woman was scheduled for emergency left atrial mass excision. Transthoracic echocardiography had revealed a large mass in the LA. Computed tomography (CT) angiography had shown normal coronaries. A $3.8 \times 3.5 / 2.9 \mathrm{~cm}$ hypodense mass was seen

\footnotetext{
${ }^{1,3}$ Professor, ${ }^{2}$ Senior Resident, ${ }^{4}$ Professor and Head

1,2,4 Department of Cardiac Anaesthesia, All India Institute of Medical Sciences, New Delhi, India

${ }^{3}$ Department of Cardiothoracic and Vascular Surgery, All India Institute of Medical Sciences, New Delhi, India

Corresponding Author: Neeti Makhija, Professor, Department of Cardiac Anaesthesia, All India Institute of Medical Sciences, New Delhi, India, Phone: +911126593423, e-mail: neetimakhija@hotmail.com
}

attached to anterior left atrial wall close to aortic annulus projecting into left ventricle (LV) in diastole. Because of the risk of systemic embolization and/or mitral valve obstruction, the patient was taken for urgent surgical resection. After induction of anesthesia, TEE examination was performed using X7-2t Philips ultrasound probe and ultrasound machine (Phillips iE33 model, Bothell, WA, USA). Two-dimensional (2D) mid-esophageal (ME) four-chamber view at sector angle of $0^{\circ}$ confirmed a mass in the LA (Fig. 1, Video 1). There was dynamic left ventricular inflow obstruction during diastole. But the mitral valve appeared intact. Assessment of LA mass for its attachment sites and extension into other chambers was done in other views. A 2D and three-dimensional (3D) ME aortic valve long-axis view at sector angle of $135^{\circ}$ revealed the mass apparently attached via broad base to the atrial surface of anterior mitral valve leaflet, aortic annulus, and adjacent LA wall, but stalk of the myxoma could not be appreciated (Fig. 2, Video 2). Thereafter, assessment was performed in transgastric and deep transgastric views. A 2D and 3D deep transgastric longaxis view at sector angle of $0^{\circ}$ revealed that the mass was attached via a broad stalk at the junction of mitral valve annulus close to AML and adjacent LA wall (Fig. 3, Video 3). The appearance was consistent with atrial myxoma. After institution of cardiopulmonary bypass, aortic cross-clamp application, and diastolic arrest, the

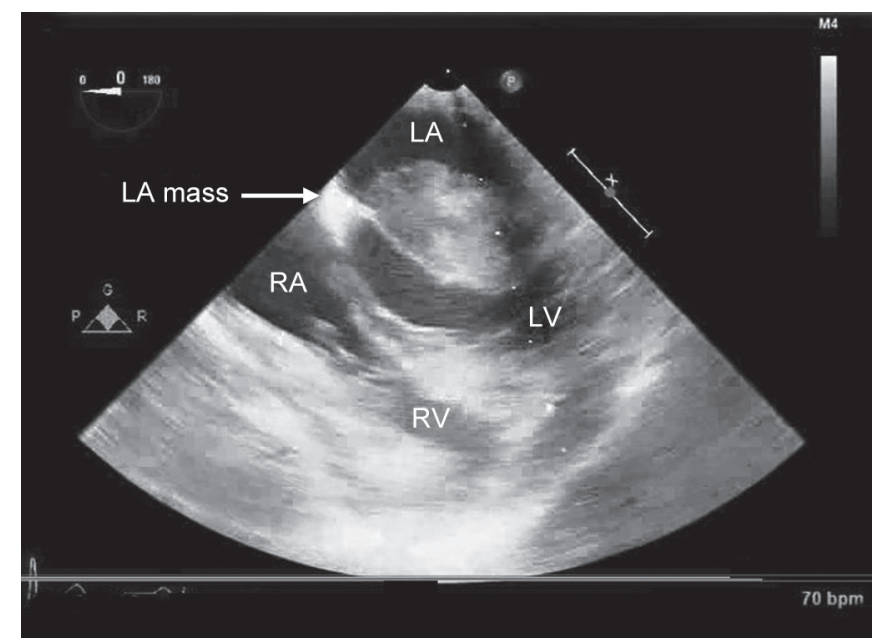

Fig. 1: A two-dimensional mid-esophageal four-chamber view at sector angle of $0^{\circ}$ showing a mass in the LA. LA: Left atrium; LV: Left ventricle; RA: Right atrium; RV: Right ventricle 


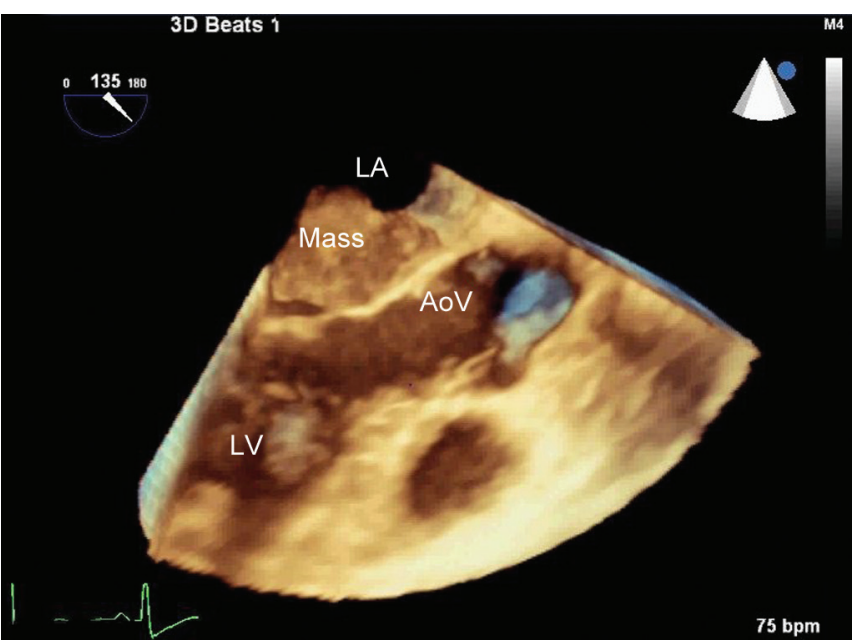

Fig. 2: A three-dimensional mid-esophageal aortic valve longaxis view at sector angle of $135^{\circ}$ revealed the mass apparently attached via broad base to the atrial surface of anterior mitral valve leaflet, aortic annulus, and adjacent left atrial wall. LA: Left atrium; LV: Left ventricle; AoV: Aortic valve

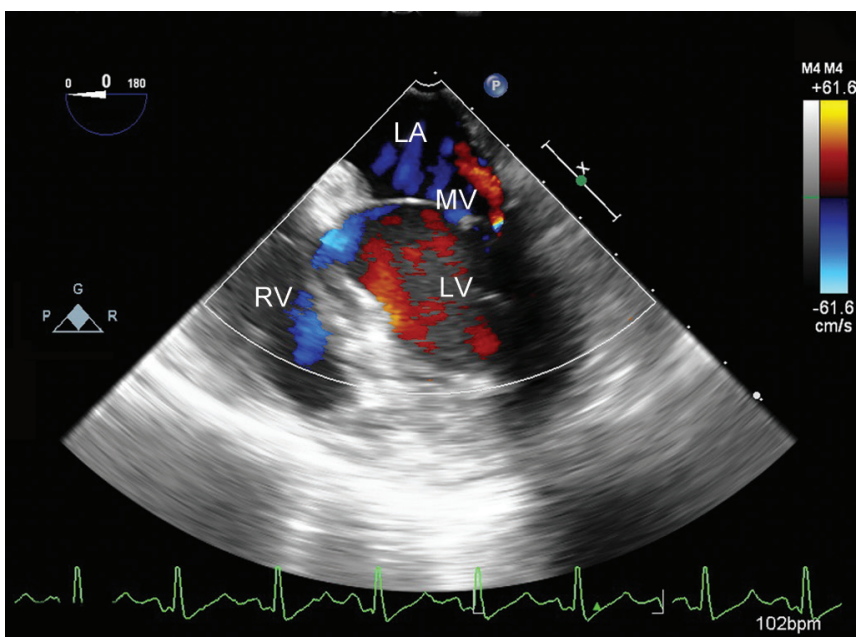

Fig. 4: A two-dimensional mid-esophageal four-chamber view at sector angle of $0^{\circ}$ showing no remnants of mass in the LA and trivial mitral regurgitation. LA: Left atrium; LV: Left ventricle; $\mathrm{RV}$ : Right ventricle; MV: Mitral valve

LA was approached through right atrium via incision at interatrial septum. A $4 \times 4 \mathrm{~cm}$ myxomatous mass was seen attached to the LA wall and adjacent annulus of mitral valve close to AML via a broad stalk. Interatrial septum was free from the mass. The mitral valve leaflets were intact. The left atrial mass was excised and Dacron patch closure of interatrial septum was done. The histological examination confirmed the diagnosis of atrial myxoma. Postoperative echocardiography in ME four-chamber view at sector angle of $0^{\circ}$ showed there were no remnants of myxoma, no mitral regurgitation, an intact atrial septal patch, and good biventricular systolic function (Figs 4 and 5). The postoperative period was uneventful, and she was discharged from hospital on the 5 th postoperative day.

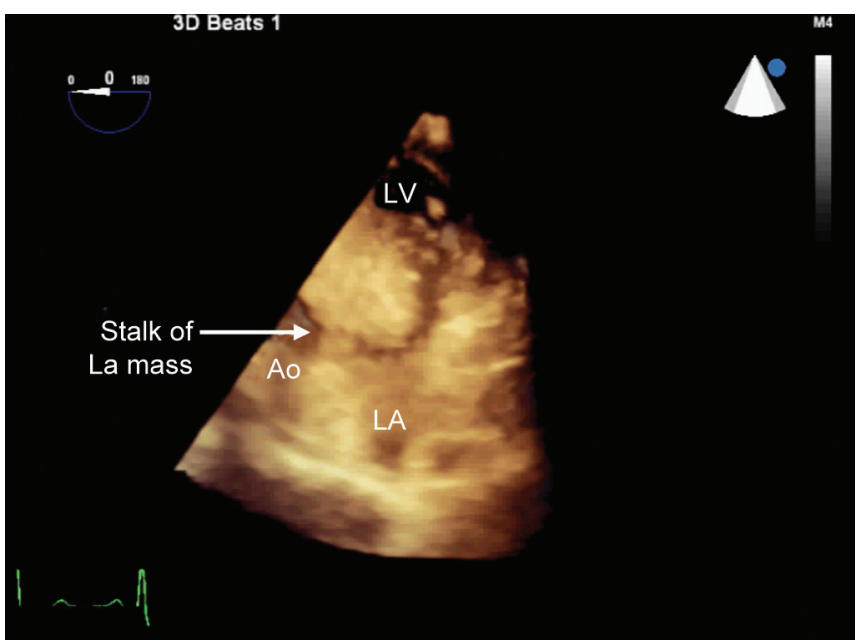

Fig. 3: A three-dimensional deep transgastric long-axis view at sector angle of $0^{\circ}$ showing mass attached via a broad stalk at the junction of mitral valve annulus close to anterior mitral leaflet and adjacent left atrial wall. LA: Left atrium; LV: Left ventricle; Ao: Aorta

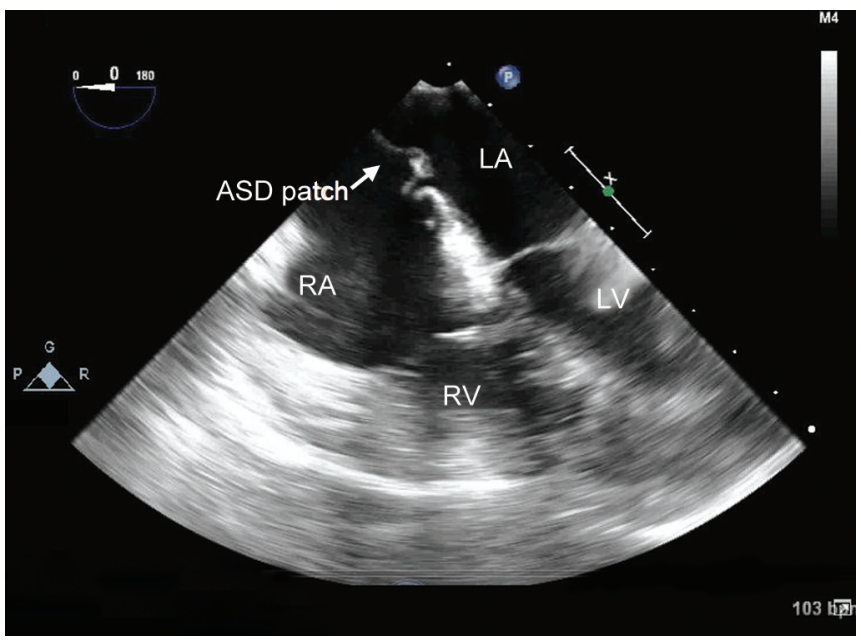

Fig. 5: A two-dimensional mid-esophageal four-chamber view at sector angle of $0^{\circ}$ showing an intact atrial septal patch. LA: Left atrium; LV: Left ventricle; RA: Right atrium; RV: Right ventricle; ASD: Atrial septal defect

\section{DISCUSSION}

Myxoma is the most frequent primary cardiac tumor. About $75 \%$ of myxomas originate in the LA, while 15 to $20 \%$ of them are located in the right atrium. However, myxomas can be found in ventricles, superior vena cava, or pulmonary veins. They have female preponderance, usually presenting in the 4th to 7 th decades. About 7 to $10 \%$ of cases run in families. Left atrial myxoma are usually attached to the interatrial septum close to fossa ovalis. Although the commonest site of origin is the interatrial septum, there are reports of its origin from other sites, namely, $\mathrm{AML}^{1}$ and left atrial appendage (LAA). ${ }^{2}$ In our patient, it was attached at unusual site at the junction of LA wall and adjacent mitral valve annulus close to AML. This site of attachment was also 
close to the aortic valve annulus since anterior leaflet of mitral valve shares the same fibrous attachment as the aortic valve.

The left atrial myxomas produce obstructive, constitutional, and embolic symptoms. The location and size of myxomas determines the clinical presentation of intracardiac blood flow obstruction, particularly the diastolic mitral inflow. The symptoms vary from dyspnea or syncope to sudden death due to complete obstruction. $^{3}$

Echocardiographic features of myxoma are globular, smooth-surfaced or irregular, friable surface with multilobular appearance, 4 to $8 \mathrm{~cm}$ in size, heterogeneous echogenicity with areas of echolucency, and sometimes calcifications.

Left atrial myxoma has to be differentiated from other masses in the LA, the most common being a thrombus. Other uncommon masses that may be present include adenocarcinoma of the lung, malignant fibrous histiocytoma, large cell lymphoma, metastatic sarcoma, and melanoma. Based on CT imaging, Scheffel et al ${ }^{4}$ concluded that the atrial myxomas and thrombi can be differentiated by their distinguishing features of size, origin, shape, mobility, and prolapse. Despite the availability of several imaging modalities, it may still be difficult to distinguish between them. Hence echocardiography plays a key role in establishing the diagnosis of patients with cardiac myxomas and thrombi. The differentiation between myxomas and thrombi is important because of the distinct treatment strategy. As far as left atrial thrombi are concerned, they are classically found in the body of the LA. In some cases, atrial thrombi may have stalk and can be misdiagnosed as myxoma and subsequently unnecessary surgical resection. Atrial masses preferably in LA are more likely to be a thrombus in presence of atrial fibrillation, enlarged atrial chamber, stenotic or prosthetic mitral and tricuspid valves, low cardiac output state, or spontaneous atrial contrast echoes. Jang et $\mathrm{l}^{5}$ suggested that in a left atrial mass with stalk, differential diagnosis between thrombus and myxoma may be difficult, and when the differential diagnosis is difficult and thrombus is a possibility, a trial of anticoagulation is advisable.

Transthoracic echocardiography has less specificity than TEE. Transesophageal echocardiography has nearly $100 \%$ sensitivity for cardiac myxoma. ${ }^{6}$ Left atrial appendage is better evaluated on TEE compared to TTE, and 2D echocardiography is the primary method for diagnosis. In our case, though preoperative TTE localized the tumor arising from the LA, but it failed to give information regarding the attachment of LA mass. Intraoperative TEE delineated the precise attachment of the tumor mass to the LA. Evidently, we could identify the stalk of LA mass by imaging in transgastric TEE views only and with 3D it could be visualized with clarity. In addition, postrepair assessment for complete removal of the tumor mass could also be performed, as well as evaluation of mitral valve for any regurgitation, and any residual shunt across the atrial septal patch could also be done.

Recurrence of cardiac myxoma is a rare condition, although it is more frequent in familial ones, observed in about $3 \%$ of patients. ${ }^{7}$ Incomplete resection of the myxoma, the proliferation of a second tumor focus, or the original tumor having an intracardiac base have all been proposed as an explanation for recurrence. Turhan et $\mathrm{al}^{2,8}$ reported case of nonfamilial solitary cardiac myxoma which, following successful resection of the primary tumor mass from the LA, recurred on the atrial surface of anterior leaflet of the mitral valve. Hence these patients should be under follow-up for few years following the first successful resection of a left atrial myxoma. Individuals undergoing surgical resection of Carneycomplex-related cardiac myxomas are at relatively high risk of recurrence. ${ }^{9}$ As myxoma in the LAA are better visualized by TEE, Turhan et $\mathrm{al}^{2}$ have suggested TEE evaluation for follow-up in cases of multiple myxomas for early detection of recurrences in the LAA.

\section{CONCLUSION}

This case highlights the unusual site of attachment of left atrial myxoma. The site of attachment was via a broad stalk at the junction of mitral valve annulus close to AML and adjacent LA wall. Transesophageal echocardiography helped in the detection of site of attachment as well as in the postoperative evaluation.

\section{REFERENCES}

1. Chen MY, Wang JH, Chao SF, Hsu YH, Wu DC, Lai CP. Cardiac myxoma originating from the anterior mitral leaflet. Jpn Heart J 2003 May;44(3):429-434.

2. Turhan S, Kilickap M, Candemir B, Berkalp B, Eren NT, Akgun $G$. Three unusual myxomas originating from the left atrial appendage: A case report. J Am Soc Echocardiogr 2005 Jun;18(6):694.

3. Gabe ED, Rodriguez Correa C, Vigliano C, San Martino J, Wisner JN, Gonzalez P, Boughen RP, Torino A, Suarez LD. Cardiac myxoma. Clinical-pathological correlation. Rev Esp Cardiol 2002 May;55(5):505-513.

4. Scheffel H, Baumueller S, Stolzmann P, Leschka S, Plass A, Alkadhi $\mathrm{H}$, Schertler T. Atrial myxomas and thrombi: comparison of imaging features on CT. AJR Am J Roentgenol 2009 Mar;192(3):639-645.

5. Jang KH, Shin DH, Lee C, Jang JK, Cheong S, Yoo SY. Left atrial mass with stalk: Thrombus or myxoma? J Cardiovasc Ultrasound 2010 Dec;18(4):154-156. 
6. Oliver, WC; Nuttall, GA. Uncommon cardiac diseases. In: Kaplan, JA., editor. Kaplan's cardiac anesthesia. 5th ed. Philadelphia: Elsevier Saunders; 2006. p. 767.

7. Carvalho MS, Andrade MJ, Abecasis J, Gouveia R, Branco L, Neves JP, Mendes M. Understanding cardiac myxoma recurrence: a case report. Rev Port Cardiol 2013 Mar;32(3): 239-242.
8. Sadeghi N, Sadeghi S, Karimi A. Mitral valve recurrence of a left atrial myxoma. Eur J Cardiothorac Surg 2002 Mar;21(3): 568-573.

9. Guenther F, Siepe M, Schlensak C, Aumann K, Anton A, Niesen WD, Markfeld-Erol F, Bode C, Geibel A. Recurrence of a familial giant multilocular cardiac myxoma in a patient with Carney's complex. Circulation 2011 Mar 1;123(8):929-932. 
